\title{
Remarks on the QCD-electroweak phase transition in a supercooled universe
}

\author{
Dietrich Bödeker॰* \\ Fakultät für Physik, Universität Bielefeld, 33501 Bielefeld, Germany
}

(Received 2 September 2021; accepted 1 November 2021; published 7 December 2021)

\begin{abstract}
In extensions of the Standard Model with no dimensionful parameters the electroweak phase transition can be delayed to temperatures of order $100 \mathrm{MeV}$. Then the chiral phase transition of QCD can proceed with six massless quarks. The top-quark condensate destabilizes the Higgs potential through the Yukawa interaction, triggering the electroweak transition. Based on the symmetries of massless QCD, it has been argued that the chiral phase transition is first order. We point out that the top-Higgs Yukawa interaction is nonperturbatively large at the QCD scale, and that its effect on the chiral phase transition may not be negligible, violating some of the symmetries of massless QCD. The remaining symmetries indicate that top quarks condense in a second-order phase transition, but top condensation might also be triggered by a firstorder symmetry-breaking transition in the light-quark sector.
\end{abstract}

DOI: 10.1103/PhysRevD.104.L111501

\section{INTRODUCTION}

First-order phase transitions are interesting for cosmology because they can leave observable relics such as the baryon asymmetry of the Universe [1,2], gravitational waves [3], or large-scale magnetic fields [4]. In the Standard Model there is neither an electroweak, nor a QCD, or quark-hadron phase transition: both the electroweak symmetry $[5,6]$ and the approximate symmetries of QCD get broken in a smooth crossover $[7,8]$.

However, in nearly scale-invariant extensions of the Standard Model there can be a first-order electroweak phase transition and, furthermore, it can have very peculiar features: The decay rate of the metastable high-temperature phase can be tiny down to very low temperatures, so that the Universe supercools well below the critical temperature [9-12]. Important examples are Coleman-Weinberg-type models [13], which contain no (negative) mass squared term for the Higgs field(s). The thermal mass squared for the Higgs field $H$ is positive, so that the state with $H=0$ remains metastable until $T=0$. In some cases the temperature becomes as low as $O(100) \mathrm{MeV}$ while the electroweak symmetry is still unbroken, and all six quark flavors are still massless. QCD with six massless quarks is expected to have a first-order chiral phase transitions [14], in which quark condensates $\langle\bar{q} q\rangle \neq 0$ form at a

\footnotetext{
*bodeker@physik.uni-bielefeld.de
}

Published by the American Physical Society under the terms of the Creative Commons Attribution 4.0 International license. Further distribution of this work must maintain attribution to the author(s) and the published article's title, journal citation, and DOI. Funded by SCOAP ${ }^{3}$. critical temperature of about $85 \mathrm{MeV}$ [15]. If this also happens in the strongly supercooled universe, the top condensate would give rise to a linear term in the effective Higgs potential through the top Yukawa interaction

$$
\mathcal{L}_{t \text {-Yukawa }}=y_{t} \bar{Q}_{3} \tilde{H} t_{R}+\text { H.c. },
$$

where $\bar{Q}_{3}=\left(\overline{t_{L}}, \overline{b_{L}}\right)$ is the doublet of third-family lefthanded quarks, $t_{R}$ is the right-handed top quark, and $\tilde{H}=i \sigma^{2} H^{*}$. The linear term destabilizes the minimum of the effective potential at $H=0$ and triggers electroweak symmetry breaking [9]. After the top condensation the universe either evolves into a metastable state, followed by another phase transition, or it could directly roll to the minimum of the effective potential. Similarly, patterns arise in other scale invariant extension, such as Randall-Sundrum models [16-18].

At a QCD phase transition the QCD coupling is large, and perturbation theory cannot be applied. Nonperturbative lattice computations with light quarks are very difficult (see $[19,20]$ for simulations with large number of flavors). Insights into the nature of a phase transition can be gained from symmetry considerations. The Lagrangian of massless QCD with six quarks is invariant under chiral $\mathrm{SU}(6)_{L} \times$ $\mathrm{SU}(6)_{R}$ flavor transformations. A quark condensate breaks the axial subgroup, leaving a vector $\mathrm{SU}(6)$ symmetry. If this happens in a second-order phase transition, then at the critical temperature some fields become effectively massless, ${ }^{1}$ and the corresponding effective theory becomes scale invariant. Then the renormalization group for the couplings of this effective theory has an infrared-stable fixed point.

\footnotetext{
${ }^{1}$ Or, equivalently, some correlations lengths diverge.
} 
The nonexistence of an infrared-stable fixed point, on the other hand, is an indication that the phase transition is first order. Near a second-order phase transition, the effective long-distance degrees of freedom are scalar fields describing the quark condensates. The effective Lagrangian, and thus the beta functions are determined by the symmetries. The beta functions can be computed in the so-called $\epsilon$ expansion: They are treated perturbatively near $d=4$ dimensions by writing $d=4-\epsilon$, and expanding in powers of $\epsilon$. The effective theory describing the long-distance degrees of freedom near a second order is threedimensional, so the $\epsilon$ expansion is applied to $\epsilon=1$. For massless QCD with $N_{f} \geq 3$ quark flavors one finds no infrared-stable fixed point, which hints at a first-order chiral phase transition [14]. At nonzero, but small quark masses, a first-order phase transition is expected, even though it has not been observed in lattice simulations so far [20-23].

\section{SYMMETRIES WITH TOP-HIGGS YUKAWA INTERACTION}

Previous discussions of an electroweak phase transition triggered by top-quark condensation [11] were based on the symmetries of QCD alone. However, neither Yukawa nor electroweak gauge interactions respect the $\mathrm{SU}(6)_{L} \times$ $\mathrm{SU}(6)_{R}$ symmetry. One may assume that electroweak interactions can be neglected, but the top Yukawa coupling becomes nonperturbatively large near the QCD scale [24,25]. In Ref. [25] the running couplings from the three-loop renormalization group of the massless Standard Model are shown: the top Yukawa coupling grows as fast as the QCD coupling and blows up when the renormalization scale approaches the QCD scale parameter, $\Lambda_{\mathrm{QCD}} \cdot{ }^{2}$ Therefore, it is likely that the top Yukawa interaction in Eq. (1) would affect the chiral phase transition. When the Higgs field and the top Yukawa interaction are included, the remaining symmetry is

$$
G=\mathrm{U}(1)_{B} \times \mathrm{U}(4)_{L} \times \mathrm{U}(5)_{R} \times G_{t},
$$

where $\mathrm{U}(1)_{B}$ represents baryon number, $\mathrm{U}(4)_{L}$ acts on $u_{L}, \ldots, c_{L}, \mathrm{U}(5)_{R}$ acts on $u_{R}, \ldots, b_{R}$, and $G_{t}=\mathrm{SU}(2) \times$ $\mathrm{U}(1)$ acts like the electroweak gauge group, but only on $Q_{3}$, $t_{R}$, and $H$.

\section{PHASE TRANSITIONS}

In a chiral phase transition $G$ is broken by expectation values of

$$
\Phi_{i j} \sim \overline{q_{L i}} q_{R j} .
$$

If such a phase transition was second order, some components of $\Phi$ would become effectively massless at the critical temperature. Unlike in the $\mathrm{SU}(6)_{L} \times \mathrm{SU}(6)_{R}$ symmetric case [14], not all $\Phi_{i j}$ become massless at the same

\footnotetext{
${ }^{2}$ The Higgs self-coupling blows up as well, but it does not affect the symmetries discussed here.
}

temperature, but only those related by the symmetry $G$. Thus the breaking of $G$ might proceed in a sequence of phase transitions.

When the top condensate forms, $\Phi_{i 6}$ with $i=5,6$ (corresponding to $b$ and $t$ ) gets a nonzero expectation value. It transforms as a doublet under $\mathrm{SU}(2) .^{3}$ The longdistance effective theory is equivalent to an $\mathrm{O}(N)$ model with $N=4$, which is known to have a second-order phase transition (see e.g., [26]). Thus, one would conclude that the breaking of $G_{t}$ through top-quark condensation happens in a second-order phase transition.

However, this is not the only possibility. Coming from high temperatures, there could first be one or several of the following phase transitions involving the light-quark sector, which break different symmetries: $(i)$ when $\Phi_{i j}$ with $i=1, \ldots, 4, j=1, \ldots, 5$ gets an expectation value, or (ii) $\Phi_{i j}$ with $i=5,6, j=1, \ldots, 5$, or $(i i i) \Phi_{i 6}$ with $i=1, \ldots, 4$. Under the symmetry-group $G$ in Eq. (2), these fields transform like $\Phi \rightarrow U^{\dagger} \Phi V$ with $U \in \mathrm{U}(4)_{L}, V \in$ $\mathrm{U}(5)_{R}$ in case (i), with $U \in \mathrm{SU}(2) \subset G_{t}, V \in \mathrm{U}(5)_{R}$ in case $(i i)$. In case $\left(\right.$ iii) the field transforms like $\Phi \rightarrow U^{\dagger} \Phi$ with $U \in \mathrm{U}(4)_{L}$. Then the long-distance behavior is again described by an $\mathrm{O}(N)$ model, this time with $N=8$, which has a second-order phase transition [26]. Results applicable to cases $(i)$ and (ii) were obtained in Ref. [27], where the fixed points for complex scalar fields $\Phi=\left(\Phi_{i j}\right)$, $i=1, \ldots, M, \quad j=1, \ldots, N$, with the $\mathrm{U}(M) \times \mathrm{U}(N)-$ symmetric Lagrangian

$\mathcal{L}=\operatorname{Tr}\left(\partial_{\mu} \Phi^{\dagger}\right)\left(\partial^{\mu} \Phi\right)+r \operatorname{Tr} \Phi^{\dagger} \Phi+\frac{u}{4}\left(\operatorname{Tr} \Phi^{\dagger} \Phi\right)^{2}+\frac{v}{4} \operatorname{Tr}\left(\Phi^{\dagger} \Phi\right)^{2}$

were studied at leading order in the $\epsilon$ expansion. There it was found that there is an infrared-stable fixed point for $M N<2$, and that there are additional fixed points when $(N+M)^{2} \geq 12(M N-2)$, i.e., for $N>N_{+}$or $N<N_{-}$ with $N_{ \pm}=5 M \pm\left[24\left(M^{2}-1\right)\right]^{1 / 2}$. This indicates that there are no infrared-stable fixed points for $(M, N)=(4,5)$ and $(M, N)=(2,5)$, corresponding to case $(i)$ and $(i i)$, respectively. One would therefore conclude that in these two cases the phase transition is first order. The analysis of [27] was extended to five [28] and recently to six loops [29]. The $\epsilon$ expansion turns out to be not particularly well behaved for $\epsilon=1$. The result of a resummation performed in Ref. [28] indicate that $N_{+}$could be slightly less than five when $M=2$, so there might be an infrared-stable fixed point for $(M, N)=(2,5)$. This was confirmed in [29]. On the other hand, Refs. [27-29] all find that $N_{+}>5$ for $M=4$, indicating that there is a first-order phase transition for $(M, N)=(4,5)$.

If there is a first-order phase transition as described in the previous paragraph, there are two possibilities. The first is that in this phase transition a top condensate emerges as well, not for symmetry reasons, but because there is a discontinuous change of the properties of the system. That would mean

\footnotetext{
${ }^{3}$ It can mix with $\tilde{H}$, which has the same quantum numbers.
} 
that the top condensate forms in a first-order phase transition. Another possibility is that the top condensate forms later in a second-order transition as described above. Which of these two possibilities is realized can probably not be inferred from symmetry considerations, but only from lattice simulations of the QCD + Higgs system.

If electroweak interactions cannot be neglected, the $\Phi_{i j}$ are not gauge invariant, and cannot serve as an order parameter indicating the breaking of a global symmetry. In particular, there is no global symmetry which gets broken when $\bar{t} t$ gets an expectation value from which one could infer information about the existence or the order of a phase transition. ${ }^{4}$ The baryon number is violated due to

\footnotetext{
${ }^{4}$ This situation is similar to the breaking of the electroweak gauge symmetry through the Higgs expectation value, and for the measured value of the Higgs boson there is no phase transition in the Standard Model.
}

electroweak sphalerons. A remaining global symmetry is $\mathrm{SU}(2)_{Q} \times \mathrm{SU}(2)_{u} \times \mathrm{SU}(3)_{d}$ acting on the left-handed quark doublets and the right-handed up-type quarks of the first two families, and on all right-handed down-type quarks. This symmetry can be broken by expectation values of the gauge invariant operators $\bar{Q}_{\alpha} \tilde{H} u_{j}(\alpha=1,2 ; j=1$, $2)$, or $\bar{Q}_{\alpha} H d_{j}(\alpha=1,2 ; j=1,2,3)$. In a phase transition in which these operators acquire an expectation value the Higgs field would also have to become nonzero. Then it would still have to overcome the barrier of the effective potential, without assistance from the top quark.

\section{ACKNOWLEDGMENTS}

I would like to thank Frithjof Karsch, Rob Pisarski, Laura Sagunski, Wolfgang Unger, and Clemens Werthmann for useful discussions or remarks. This work was funded in part by the Deutsche Forschungsgemeinschaft (DFG, German Research Foundation)—Project No. 315477589_-TRR 211.
[1] A. D. Sakharov, Violation of $C P$ invariance, C asymmetry, and baryon asymmetry of the Universe, Pis'ma Zh. Eksp. Teor. Fiz. 5, 32 (1967).

[2] V. A. Kuzmin, V. A. Rubakov, and M. E. Shaposhnikov, On the anomalous electroweak baryon number nonconservation in the early Universe, Phys. Lett. 155B, 36 (1985).

[3] E. Witten, Cosmic separation of phases, Phys. Rev. D 30, 272 (1984).

[4] T. Vachaspati, Magnetic fields from cosmological phase transitions, Phys. Lett. B 265, 258 (1991).

[5] K. Kajantie, M. Laine, K. Rummukainen, and M.E. Shaposhnikov, Is There a Hot Electroweak Phase Transition at $\mathrm{m}(\mathrm{H})$ Larger or Equal to $\mathrm{m}(\mathrm{W})$ ?, Phys. Rev. Lett. 77, 2887 (1996).

[6] F. Csikor, Z. Fodor, and J. Heitger, Endpoint of the Hot Electroweak Phase Transition, Phys. Rev. Lett. 82, 21 (1999).

[7] Y. Aoki, G. Endrodi, Z. Fodor, S. D. Katz, and K. K. Szabo, The Order of the quantum chromodynamics transition predicted by the standard model of particle physics, Nature (London) 443, 675 (2006).

[8] H. T. Ding et al. (HotQCD Collaboration), Chiral Phase Transition Temperature in $(2+1)$-Flavor QCD, Phys. Rev. Lett. 123, 062002 (2019).

[9] E. Witten, Cosmological consequences of a light Higgs boson, Nucl. Phys. B177, 477 (1981).

[10] J. R. Espinosa and M. Quiros, Novel effects in electroweak breaking from a hidden sector, Phys. Rev. D 76, 076004 (2007).

[11] S. Iso, P. D. Serpico, and K. Shimada, QCD-Electroweak First-Order Phase Transition in a Supercooled Universe, Phys. Rev. Lett. 119, 141301 (2017).
[12] V. Brdar, A. J. Helmboldt, and M. Lindner, Strong supercooling as a consequence of renormalization group consistency, J. High Energy Phys. 12 (2019) 158.

[13] S. R. Coleman and E. J. Weinberg, Radiative corrections as the origin of spontaneous symmetry breaking, Phys. Rev. D 7, 1888 (1973).

[14] R. D. Pisarski and F. Wilczek, Remarks on the chiral phase transition in chromodynamics, Phys. Rev. D 29, 338 (1984).

[15] J. Braun and H. Gies, Chiral phase boundary of QCD at finite temperature, J. High Energy Phys. 06 (2006) 024.

[16] G. Servant, Baryogenesis from Strong $C P$ Violation and the QCD Axion, Phys. Rev. Lett. 113, 171803 (2014).

[17] B. von Harling and G. Servant, QCD-induced electroweak phase transition, J. High Energy Phys. 01 (2018) 159.

[18] P. Baratella, A. Pomarol, and F. Rompineve, The supercooled Universe, J. High Energy Phys. 03 (2019) 100.

[19] M. P. Lombardo, K. Miura, T. J. Nunes da Silva, and E. Pallante, One, two, zero: Scales of strong interactions, Int. J. Mod. Phys. A 29, 1445007 (2014).

[20] F. Cuteri, O. Philipsen, and A. Sciarra, On the order of the QCD chiral phase transition for different numbers of quark flavours, arXiv:2107.12739.

[21] P. de Forcrand and M. D'Elia, Continuum limit and universality of the Columbia plot, Proc. Sci. LATTICE2016 (2017) 081 [arXiv:1702.00330].

[22] O. Philipsen, Constraining the phase diagram of QCD at finite temperature and density, Proc. Sci. LATTICE2019 (2019) 273 [arXiv:1912.04827].

[23] Y. Kuramashi, Y. Nakamura, H. Ohno, and S. Takeda, Nature of the phase transition for finite temperature $N_{\mathrm{f}}=3$ 
QCD with nonperturbatively $\mathrm{O}(a)$ improved Wilson fermions at $N_{\mathrm{t}}=12$, Phys. Rev. D 101, 054509 (2020).

[24] R. A. Flores and M. Sher, Is Coleman-Weinberg symmetry breaking in the standard model ruled out?, Nucl. Phys. B238, 702 (1984).

[25] T. Hambye, A. Strumia, and D. Teresi, Super-cool dark matter, J. High Energy Phys. 08 (2018) 188.

[26] J. Zinn-Justin, Quantum field theory and critical phenomena, Int. Ser. Monogr. Phys. 92, 1 (1996).
[27] R. D. Pisarski and D. L. Stein, Critical behavior of linear $\phi^{4}$ models With $G \times G^{\prime}$ symmetry, Phys. Rev. B 23, 3549 (1981).

[28] P. Calabrese and P. Parruccini, Five loop epsilon expansion for $U(n) \times U(m)$ models: Finite temperature phase transition in light QCD, J. High Energy Phys. 05 (2004) 018.

[29] L. T. Adzhemyan, E. V. Ivanova, M. V. Kompaniets, A. Kudlis, and A. I. Sokolov, Six-loop $\varepsilon$ expansion of threedimensional $\mathrm{U}(n) \times \mathrm{U}(m)$ models, arXiv:2104.12195. 